\title{
On the blow-up criterion for the Hall-MHD problem with partial dissipation in $\mathrm{R}^{\wedge} 3$
}

\author{
Baoying $\mathrm{Du}^{1}$ \\ ${ }^{1}$ Yibin University
}

September 24, 2021

\begin{abstract}
We deal with the incompressible 3D Hall-magnetohydrodynamics with partial dissipation, a new blow-up criterion is obtained, based on which we also prove a new global solutions with small data.

\section{Hosted file}

On the blow-up criterion for the Hall-MHD problem with partial dissipation in R3.pdf available at https://authorea.com/users/435337/articles/538436-on-the-blow-up-criterion-forthe-hall-mhd-problem-with-partial-dissipation-in-r-3
\end{abstract}

